See discussions, stats, and author profiles for this publication at: https://www.researchgate.net/publication/341592022

\title{
Molecular Plant Responses to Combined Abiotic Stresses Put a Spotlight on Unknown and Abundant Genes
}

Article in Journal of Experimental Botany · May 2020

DOI: $10.1093 / \mathrm{jxb} /$ eraa250

CITATIONS

5

6 authors, including:

Nasser Sewelam

Faculty of Science, Tanta University

16 PUBLICATIONS 1,463 CITATIONS

SEE PROFILE

2. Veronica G Maurino

University of Bonn

108 PUBLICATIONS 3,136 CITATIONS

SEE PROFILE
READS

238

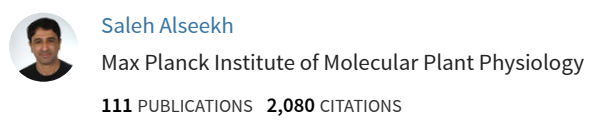

SEE PROFILE

Some of the authors of this publication are also working on these related projects:

"Involvement of 2-hydroxy acids in plant metabolism" and "Effects of organellar originated-H2O2 in signalling and biotic interactions" View project 


\title{
Molecular plant responses to combined abiotic stresses put a spotlight on unknown and abundant genes
}

\author{
Nasser Sewelam ${ }^{1,2}$, Dominik Brilhaus ${ }^{3}$, Andrea Bräutigam ${ }^{4}$, Saleh Alseekh ${ }^{5,6}$, Alisdair R. Fernie ${ }^{5,6}$ and \\ Veronica G. Maurino $0^{1,7, *}$ \\ 1 Institute of Developmental and Molecular Biology of Plants, and Cluster of Excellence on Plant Sciences (CEPLAS), Heinrich Heine \\ University Düsseldorf, Universitätsstraße 1, 40225 Düsseldorf, Germany \\ 2 Botany Department, Faculty of Science, Tanta University, 31527, Tanta, Egypt \\ ${ }^{3}$ Institute of Plant Biochemistry, and Cluster of Excellence on Plant Sciences (CEPLAS), Heinrich Heine University Düsseldorf, \\ Universitätsstraße 1, 40225 Düsseldorf, Germany \\ ${ }^{4}$ Computational Biology, Faculty of Biology, Bielefeld University, D-33615 Bielefeld, Germany \\ ${ }^{5}$ Max-Planck-Institute of Molecular Plant Physiology, Am Mühlenberg 1, 14476 Potsdam-Golm, Germany \\ ${ }^{6}$ Center for Systems Biology and Biotechnology, 4000 Plovdiv, Bulgaria \\ 7 Department of Molecular Plant Physiology, Institute of Molecular Physiology and Biotechnology of Plants, University of Bonn, Kirschalle \\ 1, 53115 Bonn, Germany \\ * Correspondence: vero.maurino@uni-bonn.de
}

Received 6 March 2020; Editorial decision 14 May 2020; Accepted 19 May 2020

Editor: Christine Foyer, University of Birmingham, UK

\begin{abstract}
Environmental stresses such as drought, heat, and salinity limit plant development and agricultural productivity. While individual stresses have been studied extensively, much less is known about the molecular interaction of responses to multiple stresses. To address this problem, we investigated molecular responses of Arabidopsis to single, double, and triple combinations of salt, osmotic, and heat stresses. A metabolite profiling analysis indicated the production of specific compatible solutes depending on the nature of the stress applied. We found that in combination with other stresses, heat has a dominant effect on global gene expression and metabolite level patterns. Treatments that include heat stress lead to strongly reduced transcription of genes coding for abundant photosynthetic proteins and proteins regulating the cell life cycle, while genes involved in protein degradation are up-regulated. Under combined stress conditions, the plants shifted their metabolism to a survival state characterized by low productivity. Our work provides molecular evidence for the dangers for plant productivity and future world food security posed by heat waves resulting from global warming. We highlight candidate genes, many of which are functionally uncharacterized, for engineering plant abiotic stress tolerance.
\end{abstract}

Keywords: Global gene expression, global warming, metabolite profiles, multiple abiotic stresses.

\section{Introduction}

Scientific and intergovernmental reports warn of dangerous consequences of global warming for various aspects of human life, with considerable threats to plant productivity (Lobell and Field, 2007; Battisti and Naylor, 2009; Lobell et al., 2011; Deryng et al., 2014; Lesk et al., 2016; IPCC, 2018). Current temperatures are approximately $1{ }^{\circ} \mathrm{C}$ above pre-industrial 
levels; a further increase of global temperatures by $0.5{ }^{\circ} \mathrm{C}$ would increase the associated risks. An increase to $2{ }^{\circ} \mathrm{C}$ above pre-industrial temperatures might lead to the complete loss of many ecosystems (IPCC, 2018). The frequency of more extreme climatic events, such as droughts and heatwaves, will increase with additional elevations of global temperatures (IPCC, 2014). Drought and high temperatures significantly reduced cereal production by about 10\% between 1964 and 2007 (Lesk et al., 2016). The ever-increasing threat of extreme weather that is concomitant to global warming-in particular the increased occurrence of very high temperatures-might lead to catastrophic decreases in crop productivity and result in worldwide famine (Bita and Gerats, 2013).

One-third of the earth's landmass is dominated by arid and semiarid weather conditions (Huang et al. 2010). Considering the foreseen climate changes and global warming effects, these arid areas are expected to expand massively (Park et al., 2018); consequently, many crop plants are expected to suffer from a wide range of new stress combinations. Arid climate is characterized by high temperatures. These not only lead to high evaporation rates and hence cause drought, but also favor the accumulation of salts in the surface soil layers close to the root zones of crop plants, causing salinity stress. Thus, plants growing in arid areas are frequently subjected to a combination of heat, drought, and salt stresses; based on the climate and nature of the soil in a specific geographical region, these stresses may occur individually or in combination. In order to develop successful cultivation strategies that secure the world's food demands under the foreseen global warming, it is crucial to understand plant responses to complex environmental challenges (Battisti and Naylor, 2009; Lobell et al., 2011; Deryng et al., 2014).

A plethora of studies have investigated plant responses to individual stress conditions at the physiological and molecular levels (Schenk et al., 2000; Kilian et al., 2007; Matsui et al., 2008; Shaik and Ramakrishna, 2013). This type of study has significantly improved our understanding of the mechanisms of plant responses to environmental stresses. To apply this knowledge to conditions outside of the laboratory, we urgently need to elucidate the interactions that delineate plant responses to natural, multi-stress environments (Mittler, 2006; Atkinson and Urwin, 2012; Prasch and Sonnewald, 2015; Pandey et al., 2015; Zhang and Sonnewald, 2017).

Few studies exist on plant responses to combined stresses. Fortunately, several groups have started to overcome the technical issues that are encountered in designing the experimental set-up of combined stress factors under controlled conditions in the laboratory. Thus, in addition to two pioneering studies by Rizhsky et al. $(2002,2004)$ on the effects of combined heat and drought stresses on gene expression profiles in tobacco and Arabidopsis, several recent genome-wide studies have made a shift to investigate plant responses to combined stresses (Rasmussen et al., 2013; Prasch and Sonnewald, 2013; Sewelam et al., 2014; Barah et al., 2016; Georgii et al., 2017; Shaar-Moshe et al., 2017; Osthoff et al., 2019). These studies suggested that plant molecular response to different individual stresses could not be used to predict the plant responses to combined stress treatments.
Here, we investigated molecular responses of the model plant Arabidopsis to single, double, and triple combinations of salt, osmotic, and heat stresses. Our results reveal a dominant effect of heat stress over salinity and osmotic stresses with regard to the global changes in gene expression and relative metabolite levels. These findings turn more attention towards the profound effects of heatwaves that are unavoidable in the current global warming era. We found that plants experiencing combined abiotic stresses reprogram their transcriptional machinery to down-regulate the expression from the most abundant genes, most probably as a strategy to save resources for defense and survival. Relative metabolite levels determined during the single stresses and their combinations highlighted the coordinated modulation of specific amino acid levels, especially in treatments including heat stress. Furthermore, we found that different compounds are used as compatible solutes depending on the nature of the abiotic stress.

\section{Materials and methods}

\section{Experimental design and stress treatment scheme}

Seeds of wild-type Arabidopsis ecotype Columbia-0 (Col-0; WT) were sown on soil. After stratification at $4{ }^{\circ} \mathrm{C}$ for $2 \mathrm{~d}$, the seeds were transferred to a long-day ( $16 \mathrm{~h}$ light $-8 \mathrm{~h}$ dark) growth chamber using a mix of Spectralux®Plus NL 36W/840 (Radium) and Fluora L 36W/77 (Osram) bulbs with a light intensity of approximately $100 \mu \mathrm{E} \mathrm{m} \mathrm{m}^{-2} \mathrm{~s}^{-1}$ and a temperature regime of $22^{\circ} \mathrm{C}$ day $-18^{\circ} \mathrm{C}$ night temperatures. Twoweek-old seedlings were transplanted to new soil. After another $14 \mathrm{~d}$ of growth, plants were subjected to the stress treatments (Fig. 1A).

To simulate the effects of complex field environments on Arabidopsis plants, we used well-established analog artificial stress conditions. These conditions lead to low variability and allowed us to tightly control stress level and onset and to grow plants in limited space (Verslues et al., 2006; Lawlor, 2013). As a proxy for drought stress, we applied mannitol, an osmoticum that lowers the water potential of the soil, making it difficult for the plants to extract water (Verslues et al., 2006). To simulate salt stress, we watered the plants with $\mathrm{NaCl}$ (Munns and Tester, 2008). For heat stress we exposed the plants to a defined elevated temperature that does not cause heat shock in Arabidopsis. For the salt stress treatment, plants were watered with $150 \mathrm{mM} \mathrm{NaCl}$ solution $16 \mathrm{~h}$ before sampling. The osmotic stress treatment was applied by watering plants with $200 \mathrm{mM}$ mannitol $16 \mathrm{~h}$ before sampling. For the heat treatment, plant trays were transferred to a growth cabinet pre-adjusted at $35^{\circ} \mathrm{C}$ for $4 \mathrm{~h}$ before sampling. We selected a sampling time of $16 \mathrm{~h}$ after stress initiation for salt and mannitol treatments because the plant response to these treatments peaks between 12 and $24 \mathrm{~h}$. In contrast, $4 \mathrm{~h}$ was chosen for heat, as plant response to heat is reported to peak from 2 to 4 h (Kilian et al. 2007, Sewelam et al.,2014). To avoid the circadian clock effects that could interfere with the interactions between our treatments, we started the heat treatment $3 \mathrm{~h}$ after light onset, and we collected all samples at midday when the plant's metabolism is at a steady state. Double treatment of salt and mannitol stress was applied by watering plants with a mixed solution containing final concentrations of $150 \mathrm{mM} \mathrm{NaCl}$ and $200 \mathrm{mM}$ mannitol. For double treatments of heat with $\mathrm{NaCl}$ or mannitol, a fraction of the plants that were watered with $\mathrm{NaCl}$ or mannitol were moved after $12 \mathrm{~h}$ to a growth cabinet pre-adjusted at $35^{\circ} \mathrm{C}$ for an extra $4 \mathrm{~h}$, resulting in a total stress time of $16 \mathrm{~h}$ before sampling (Fig. 1B).

For multiple stress treatments, a patch of the plants treated with the double treatment of $\mathrm{NaCl} 150 \mathrm{mM}$ and $200 \mathrm{mM}$ mannitol were transferred after $12 \mathrm{~h}$ to a growth cabinet pre-adjusted at $35^{\circ} \mathrm{C}$ for an extra $4 \mathrm{~h}$, resulting in a total stress time of $16 \mathrm{~h}$ before sampling (Fig. 1A). To apply a multiple stress treatment similar to field conditions as much as possible, Arabidopsis plants were treated with salt and mannitol $1 \mathrm{~h}$ before the light was turned off, and were kept for $12 \mathrm{~h}$ before starting the 
A

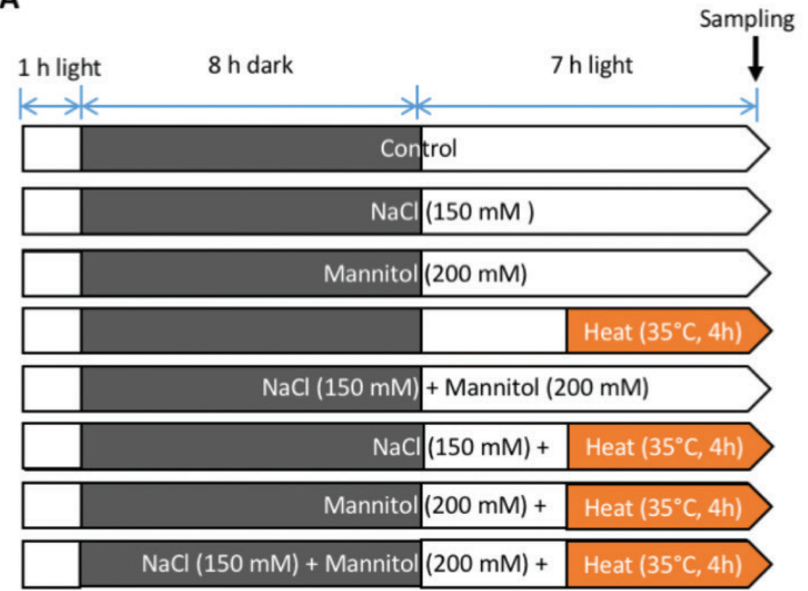

B

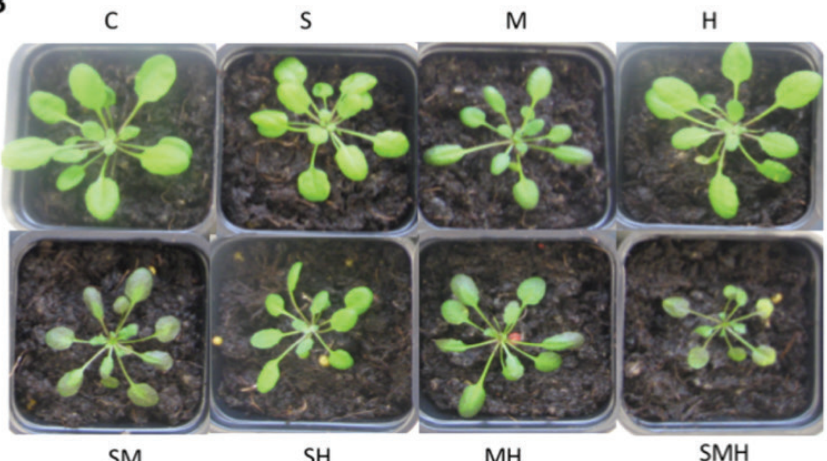

C

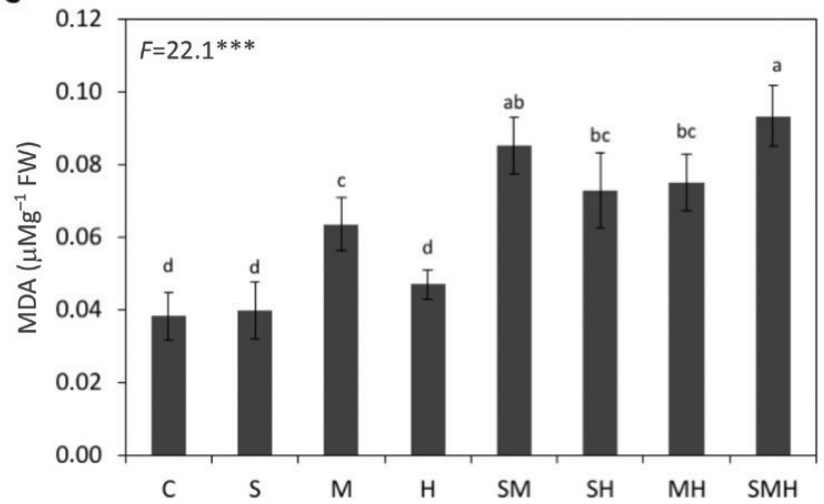

Fig. 1. (A) Scheme of the experimental design. (B) Representative Arabidopsis plants $5 \mathrm{~d}$ after the treatments in (A). (C) Malondialdehyde (MDA) content in leaves of Arabidopsis plants $5 \mathrm{~d}$ after the treatments. Values are means of three biological replicates. Error bars represent standard deviation. $F$-values represent one-way ANOVA. ${ }^{*} P<0.01$. Means with different letters are significantly different at $P<0.05$ according to the Tukey HSD test. C, control (untreated plants); $\mathrm{H}$, heat; $\mathrm{M}$, mannitol (osmotic); S, salt.

heat treatment. After $3 \mathrm{~h}$ of light, plant trays were transferred to a $35^{\circ} \mathrm{C}$ growth cabinet for an extra $4 \mathrm{~h}$, resulting in a total treatment time of $16 \mathrm{~h}$ (Fig. 1A). Rosettes were harvested and immediately frozen in liquid nitrogen. Tissue was ground into fine powder in the presence of liquid nitrogen, and then kept at $-80^{\circ} \mathrm{C}$ until use. Frozen samples were used for RNA extraction and metabolite analysis. For malondialdehyde (MDA) quantification, samples were collected $4 \mathrm{~d}$ after the application of the treatments. All experiments were performed in biological triplicates.

\section{MDA measurements}

Lipid peroxidation was measured by determining the amount of MDA. MDA concentration was estimated by the method of Heath and Packer (1968). A sample of $0.5 \mathrm{~g}$ fresh leaves was extracted in $10 \mathrm{ml} \mathrm{5 \%} \mathrm{(w/v)} \mathrm{tri-}$ chloroacetic acid and the homogenate was clarified by centrifugation at $1500 \mathrm{~g}$ for $10 \mathrm{~min}$. The supernatant $(2 \mathrm{ml})$ was mixed with $2 \mathrm{ml}$ of $0.67 \%$ $(\mathrm{w} / \mathrm{v})$ thiobarbaturic acid, incubated at $95^{\circ} \mathrm{C}$ in a water bath for $20 \mathrm{~min}$, and cooled immediately. Absorbance was read at 532 and $600 \mathrm{~nm}$. MDA concentration $\left(\mu \mathrm{M} \mathrm{g}^{-1}\right.$ fresh weight) was calculated using the extinction coefficient $155 \mathrm{mM}^{-1} \mathrm{~cm}^{-1}$. The calibration curve was constructed in the concentration range of $0.2-2 \mu \mathrm{M}$.

The results were analysed using one-way analysis of variance (ANOVA) Significant differences among the means were identified using the Tukey HSD test.

\section{RNA extraction and sequencing}

For RNA extraction, rosette leaves from wild-type plants were harvested at the sampling times indicated in Fig. 1A and frozen in liquid nitrogen. Total RNA was extracted with the SV Total RNA Isolation System (Promega, Madison, WI, USA).The integrity of RNA was analysed by gel electrophoresis, and its concentration was measured using a NanoDrop (ND-1000) spectrophotometer. Libraries were prepared using the TruSeq RNA Sample Prep Kit v2 (Illumina, San Diego, CA, USA) and quantified with a Qubit 2.0 (Thermo Fisher Scientific, Waltham, MA, USA) Samples were multiplexed with 12 libraries per lane and sequenced in paired-end mode (Rapid Run, 100 bp read length) on an Illumina HiSeq 2500 platform.

\section{Read mapping and mRNA-Seq data analysis}

After successful quality control with the Fast QC software (v0.11.5), Illumina reads were quantified by mapping against the Arabidopsis reference transcriptome (Araport 11 representative CDS, Araport11_ genes.201606.cds.fasta.gz, retrieved from www.araport.org) using Kallisto v 0.45.1 (Bray et al., 2016) in default mode with 50 times bootstrapping (option, $b$ 50). Kallisto provides normalized transcript abundance as transcripts per million (TPM). Differential transcript abundance comparing each of the treatments against the control were determined using the likelihood ratio test implemented in sleuth v0.30.0 (Pimentel et al., 2017). A significance threshold $\alpha$ of 0.05 was chosen after adjusting $P$-values (thereafter termed ' $q$-value') for multiple hypothesis testing via the Benjamini-Hochberg correction (Benjamini and Hochberg, 1995) as implemented in sleuth. The ' $b$ value' supplied by sleuth was used as adapted $\log 2$-fold change between the respective treatment and control. $k$-means clustering was done with function $k$-means in $\mathrm{R}$ choosing eight clusters $(k=8)$, as the clustering analyses that included more than eight clusters showed no new expression patterns. The inputs for $k$-means were average TPM of each treatment $(n=3)$ after filtering for significantly differentially expressed transcripts $(q \leq 0.05)$ and using a minimum foldchange threshold of 1 . All statistical analyses were performed in the R environment for statistical computing (v3.6.1 provided by the CRAN 
project; R Core Team, 2018). Gene annotation and categorization was retrieved from publicly accessible databases, including Araport (www. araport.org/data/araport11), gene ontology (GO) annotation (www. arabidopsis.org), Mapman categorization (August 2012, www.plabipd. de/portal/web/guest/mapman), transcription factors (www.plntfdb.bio. uni-potsdam.de/v3.0), and genes involved in lipid metabolism (www. aalip.plantbiology.msu.edu/downloads).

\section{Gas chromatography-mass spectrometry analysis}

Extraction and analysis by gas chromatography-mass spectrometry (GCMS) was performed using the same equipmental set-up and exact same protocol as described in Lisec et al. (2006). Briefly, frozen ground material was homogenized in $300 \mu \mathrm{l}$ of methanol at $70{ }^{\circ} \mathrm{C}$ for $15 \mathrm{~min}$ and $200 \mu \mathrm{l}$ of chloroform followed by $300 \mu \mathrm{l}$ of water was added. The polar fraction was dried under vacuum, and the residue was derivatized for $120 \mathrm{~min}$ at $37^{\circ} \mathrm{C}$ (in $40 \mu \mathrm{l}$ of $20 \mathrm{mg} \mathrm{ml}^{-1}$ methoxyamine hydrochloride (SigmaAldrich, cat. no. 593-56-6) in pyridine followed by a 30 min treatment at $37^{\circ} \mathrm{C}$ with $70 \mu \mathrm{l}$ of $\mathrm{N}$-methyl-N-(trimethylsilyl)trifluoroacetamide (MSTFA reagent; Macherey-Nagel, cat. no. 24589-78-4).An autosampler Gerstel Multi-Purpose system (Gerstel GmbH \& Co.KG, Mülheim an der Ruhr, Germany) was used to inject $1 \mu$ l of the samples in splitless mode to a chromatograph coupled to a time-of-flight mass spectrometer system (Leco Pegasus HT TOF-MS; Leco Corp., St Joseph, MI, USA). Helium was used as carrier gas at a constant flow rate of $2 \mathrm{ml} \mathrm{s}^{-1}$ and GC was performed on a $30 \mathrm{~m}$ DB-35 column (capillary column, $30 \mathrm{~m}$ length, $0.32 \mathrm{~mm}$ inner diameter, $0.25 \mu \mathrm{m}$ film thickness, PN: G42, Agilent). The injection temperature was $230^{\circ} \mathrm{C}$ and the transfer line and ion source were set to $250{ }^{\circ} \mathrm{C}$. The initial temperature of the oven $\left(85^{\circ} \mathrm{C}\right)$ increased at a rate of $15^{\circ} \mathrm{C} \min ^{-1}$ up to a final temperature of $360{ }^{\circ} \mathrm{C}$. After a solvent delay of $180 \mathrm{~s}$, mass spectra were recorded at 20 scans $\mathrm{s}^{-1}$ with $\mathrm{m} / z 70-600$ scanning range. Chromatograms and mass spectra were evaluated using Chroma TOF 4.5 (Leco) and TagFinder 4.2 software. Metabolites were annotated based on a retention index calculation with deviation $<5 \%$ and compared with the reference data of the Golm Metabolome Database, http://gmd.mpimp-golm.mpg.de (Luedemann et al., 2008). The results were statistically analysed using Student's $t$-test, using a threshold of $P<0.05$ between the samples of the control and treated plants.

\section{Heat map and principal component analysis}

Heat maps were created by $\mathrm{MeV}$ software (http://mev.tm4.org), using the $\log 2$ of the metabolite fold changes with respect to the control. Each cell in the heatmap represents the $\log 2$ average fold change value. Hierarchical clustering was applied to rows and columns using Pearson's correlation coefficient $(>0.80)$. Principal component analysis was performed with the function prcomp implemented in $\mathrm{R}$.

\section{Results}

\section{Plant phenotypes and stress level under individual and combined stress treatments}

To study the interacting effects of different combinations of environmental stresses, 4-week-old Arabidopsis plants were subjected to single, double, and triple combinations of salt, osmotic, and heat stresses as shown in Fig. 1A. The applied treatments were selected and designed to simulate the environmental conditions in arid areas, where high temperatures cause high evaporation rates, leading to water shortage and accumulation of salts. We applied mild stress doses to impose physiological changes close to those occurring in natural plant habitats.

Five days after the stress treatments, plants morphology (Fig. 1B) and the level of lipid peroxidation (Fig. 1C) were assessed. Among the single treatments, mannitol caused the highest damaging effect on plant growth and the highest lipid peroxidation level. The double treatments led to damaging effects stronger than any of the individual stresses, in which all plants showed reduced growth. After the single heat treatment, the plants recovered and grew normally, but the heat dose intensified the negative effects of salinity and osmotic stress on plant growth. Out of the double treatments the combination of salt and osmotic stresses presented the most pronounced damaging effects. The triple combined treatment caused the most sever effects compared with all other stress treatments (Fig. 1B, C).

\section{The genome-wide transcriptomic data indicate a dominant effect of heat stress}

We investigated genome-wide changes in the Arabidopsis transcriptome as a result of the interacting effects of abiotic stresses by RNA-seq. A principal component analysis (PCA) showed that the first principal component (PC1) separated the expression patterns of the treatments that include heat stress from the other treatments, explaining $46 \%$ of the gene expression variation (Fig. 2A). The second principal component (PC2), which explained an additional $13.4 \%$ of the variation, separated the patterns after treatments that include mannitol stress from all the others (Fig. 2A). Out of 27655 Arabidopsis genes represented in our study, 19244 genes were differentially expressed (up- or down-regulated; $P<0.05$ ) by at least one of the seven treatments compared with the control (see Supplementary Dataset S1 at JXB online). Comparisons of qRT-PCR data with results from genome-wide studies indicated that relative expression changes of $<2$-fold change (FC) can be unreliable (Lee et al., 2005), and thus we set a cut-off value of $\log _{2}$ of 1 , which corresponds to 2-FC in expression with regards to the control.

At a cut-off value of 2-FC we found that 3529 genes were differentially expressed by at least one stress treatment compared with control (see Supplementary Dataset S1). Stress treatments including heat significantly affected the expression of the largest numbers of genes (Fig. 2B). Heat treatment significantly induced the expression of 1107 genes and reduced the expression of 697 genes. Mannitol treatment led to the induction of expression of 229 genes, and repressed the expression of 91 genes. Salt stress induced the expression of 63 genes, and repressed the expression of 22 genes. The effects of the single stresses could be viewed at first as having synergistic effects, in which the numbers of genes induced or repressed by the combined treatments were considerably higher than those of the individual treatments. The triple combination of stresses affected the expression level of the largest number of genes, with 1222 up- and 1071 down-regulated genes (Fig. 2B).

\section{Different stress combinations cause significant reprograming of gene expression profiles: k-means clustering}

To find specific expression patterns that may untangle the interactions between different stress treatments, we clustered 
the data by $k$-means clustering over the 3529 genes that were differentially expressed $\geq 2$-FC (Fig. 3; Supplementary Table S1). We obtained eight clusters that pinpoint candidates of the abiotic stress response with potential for engineering stress-tolerant plants.

\section{Cluster 1: osmotic stress and heat have antagonistic effects on gene expression}

Cluster 1 included 281 genes, of which overall expression was not changed by salt treatment, while osmotic treatment caused induction of the majority of these genes, and heat repressed the expression of a large number of them (Fig. 3, Supplementary Table S1). This pattern suggests an antagonistic interaction of the osmotic and heat treatments. The double stress combinations show that salt treatment does not change the expression patterns induced by the individual osmotic or heat treatments. This suggests a lack of interaction between the effects of salt with either mannitol or heat on the expression profiles of the genes in this cluster. It is noteworthy that the combined osmotic and heat treatments largely reprogramed the gene expression patterns observed by the individual treatments, confirming the antagonistic interaction of these stresses on a large set of genes (120 genes, Supplementary Table S1). The triple stress treatment further validated this conclusion (Supplementary Table S1). These differences in gene expression may explain the opposing physiological effects between drought and heat (Rizhsky et al., 2002). As an example, under drought stress plants tend to close their stomata to avoid
A

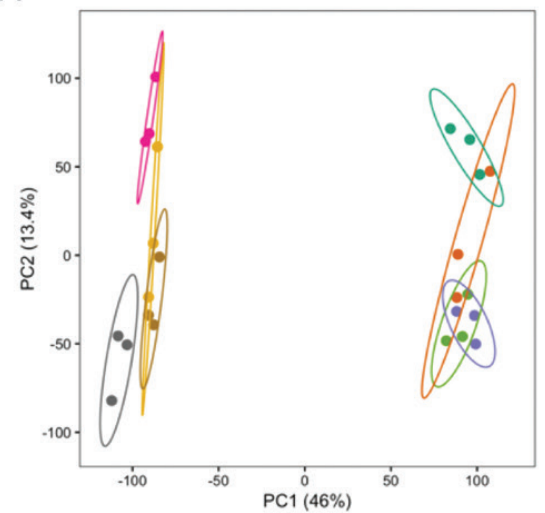

B

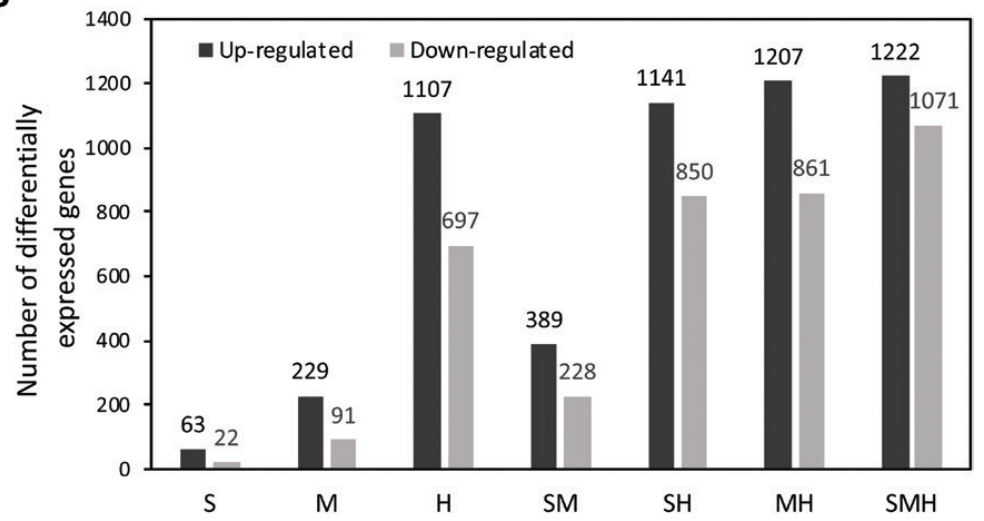

Fig. 2. (A) Principal component analysis of the RNA-Seq data. (B) Counts of differentially expressed genes at a cut-off of 2-FC. C, control; $\mathrm{H}$, heat; $\mathrm{M}$, mannitol; S, salt.
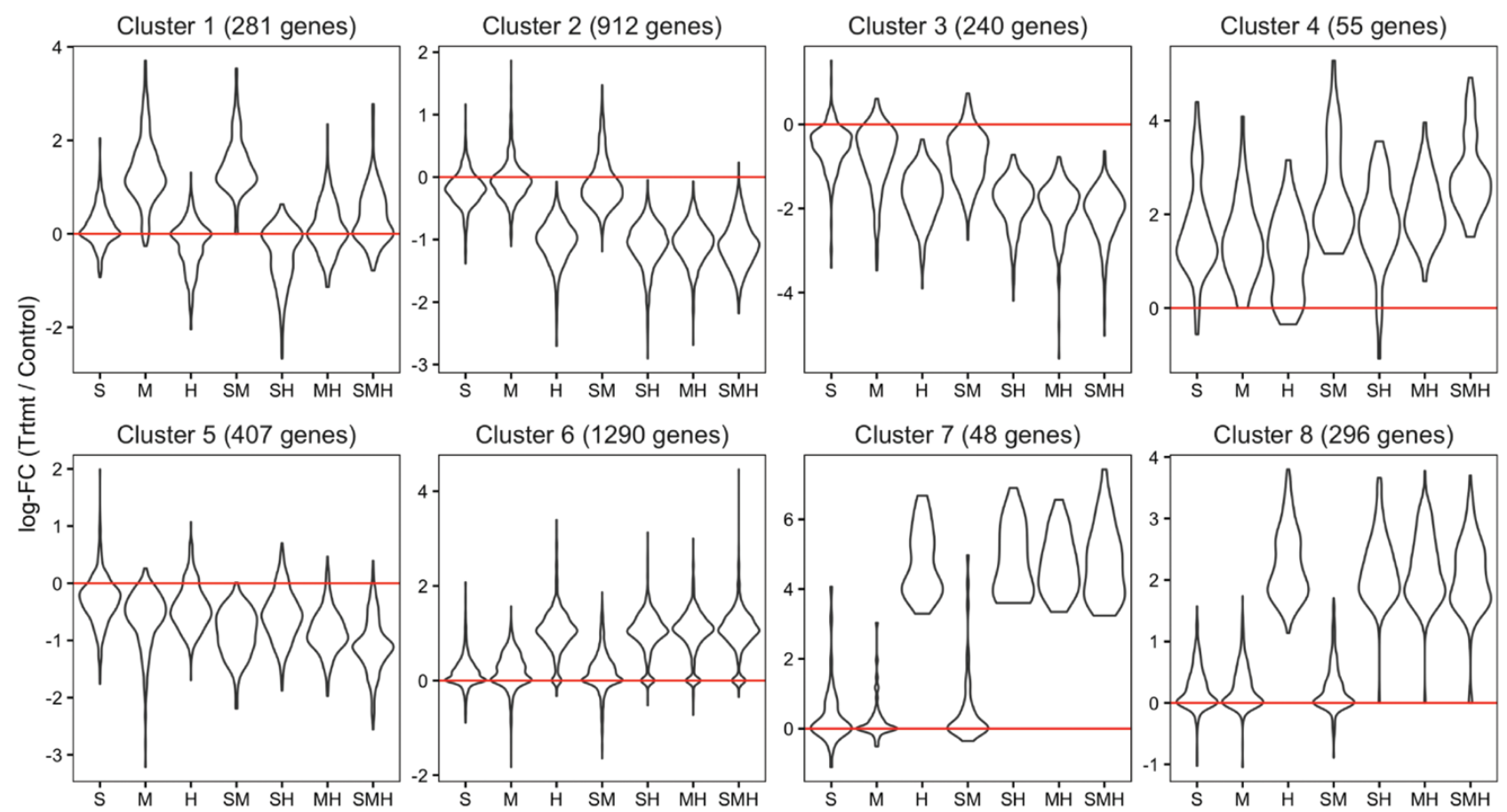

Fig. 3. $k$-Means clustering presented as a violin plot. The positive values on $y$-axis represent $\log _{2}$-fold change of induction levels, and negative values represent repression levels, while the horizontal line at 0 represents control reference. The area of the violin shape represents the proportion of genes in each cluster that had the corresponding expression level on the $y$-axis. $\mathrm{H}$, heat; $\mathrm{M}$, mannitol; $\mathrm{S}$, salt. (This figure is available in color at JXB online.) 
unnecessary water loss, while under heat conditions, stomata open to reduce leaf temperature (Rizhsky et al., 2002, 2004).

The top 50 genes antagonistically affected by osmotic and heat treatments included many genes coding for late embryogenesis abundant (LEA) proteins, receptor-like kinases (RLK), glutathione $S$-transferases and a number of WRKY transcription factors (Fig. 4A). Many of these proteins are involved in responses to drought (Goyal et al., 2005; Tunnacliffe and Wise 2007; Tolleter et al. 2010). The decline of expression of these protective genes by heat may be, at least in part, responsible for the enhanced damage observed in plants treated with the combined osmotic and heat stresses with respect to the individual conditions (Fig. 1B, C).

\section{Clusters 2, 3 and 5: a large number of genes are specifically repressed by heat}

Genes included in clusters 2 (912 genes), 3 (240 genes), and 5 (407 genes) showed repressed expression under all treatments that included heat stress (Fig. 3; Supplementary Table S1). Cluster 3 showed the most pronounced repression. Single salt and osmotic treatments and their combination had minor effects on the expression levels of genes in cluster 2, while a slight repression was observed in clusters 3 and 5 .

Cluster 2 represented about $26 \%$ of the total considered genes in our analysis. The most represented gene categories included those involved in DNA synthesis, secondary metabolism, biotic stress responses, and signaling events mediated by receptor-like kinases, calcium and G-proteins (see Supplementary Table S1). A large number of genes in this cluster (258 genes) were annotated as having unknown functions, making them candidates for further molecular analyses. Clusters 3 and 5 showed similar enrichment patterns to cluster 2. Especially cluster 5 contained many genes involved in cell wall metabolism (Supplementary Table S1), indicating that growth is negatively affected by heat.

\section{Cluster 4: a small number of genes are induced by all treatments, and salt and osmotic stresses show synergistic interactions}

Cluster 4 included a relatively small number of genes (55 genes) that were induced by all of the applied treatments (see Supplementary Table S1). Compared with the individual salt and osmotic treatments, the combined treatment of both showed a synergistic effect that changed the expression levels of most of the genes in this cluster (Fig. 3, cluster 4). Also, compared with the single and double stress treatments, the triple stress treatment caused a pronounced increase in the levels of a large number of genes. Salinity has two effects, early osmotic and relatively late ionic phases (Munns and Tester, 2008). Most probably, the genes of cluster 4 are involved in the osmotic responses of salinity as these genes are common between salt and osmotic stresses.

Many genes included in cluster 4 are annotated as involved in desiccation tolerance. Examples include AT5G06760 (LEA4-5), AT1G52690 (LEA7), AT3G02480 (a LEA, ABAinducible), AT3G17520 (a LEA, ABA-inducible), AT5G15960 (osmotic and ABA-inducible), AT2G47770 (TPSO, ABAinducible), and AT5G66400 (RAB18, dehydrin family protein) (see Supplementary Table S1). Genes in cluster 4 were highly induced by the triple combination of stresses (Fig. 3, cluster 4), and thus represent excellent candidates for engineering programs that aim to produce tolerant plants against multiple stresses.

\section{Clusters 6, 7, and 8: almost half of the differentially expressed genes, which include many oxidative phosphorylation components, are specifically induced by treatments including heat}

Genes in clusters 6,7 and 8 were specifically up-regulated by treatments with a heat stress component (Fig. 3, see Supplementary Table S1). The only difference between these three clusters was the degree of the effect. Cumulatively, these three clusters included 1634 genes that represented more than $46 \%$ of the total considered genes in our clustering analysis. A large number of these genes (around 25\%) are of unknown function; others are mostly involved in protein degradation or encode HSPs (Supplementary Table S1).

We found that 71 genes out of the 122 genes of the Arabidopsis mitochondrial genome were specifically induced by heat treatment and its combinations (see Supplementary Table S1). Most of these genes encode for subunits of complex I, cytochrome oxidase, and the ATPase,

Heat shock transcription factors and heat shock proteins are mainly induced by heat and combinations of stresses including heat

Heat shock transcription factors (HSFs) and HSPs represent master regulatory and protective elements involved in the response to heat stress. Many genes coding for these proteins have already been incorporated in molecular breeding programs for sustainable agriculture (Ritossa, 1962; Swindell et al., 2007; Scharf et al., 2012; Niu and Xiang, 2018). As our transcriptome analysis showed that heat has a dominant effect on global gene expression, we specifically analysed the expression patterns of all annotated Arabidopsis HSFs (23 genes) and HSPs (156 genes) included in our study (see Supplementary Table S2) under all stress treatments.

We found that 11 HSFs were significantly induced by treatments including heat stress (Fig. 4B). In addition, HSFA7A was significantly repressed by salt and osmotic stresses. Notably, HSFA $6 A$ was strongly induced by all treatments that included salinity and osmotic stress. HSFA6B showed specific induction by the triple combinations of stresses. Three HSF genes, HSFA1E, HSF3, and HSFA5, were repressed by treatments including a heat stress component. The remaining four HSF genes, HSFB3, HSFA1A, HSFA9, and AT4G18870, showed no responsiveness to any of the applied stress treatments (Fig. 4B). The HSF genes that were repressed by heat and/or other abiotic stress represent interesting targets for functional characterization not only to provide new insights into the plant response to abiotic stresses, but also for their promising incorporation in molecular breeding programs to enhance plant fitness.

In our analysis we also found that most members of all HSP families-in particular sHSPs and HSP70-were specifically induced by treatments that included heat stress (Fig. 4C), indicating that high temperature is the most severe 
A

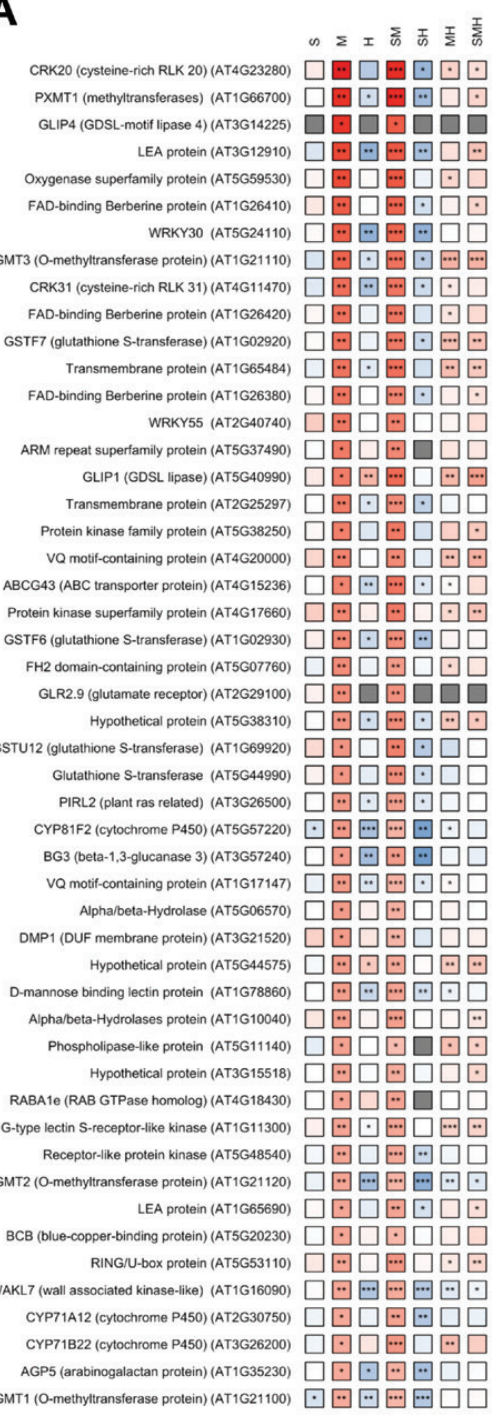

C

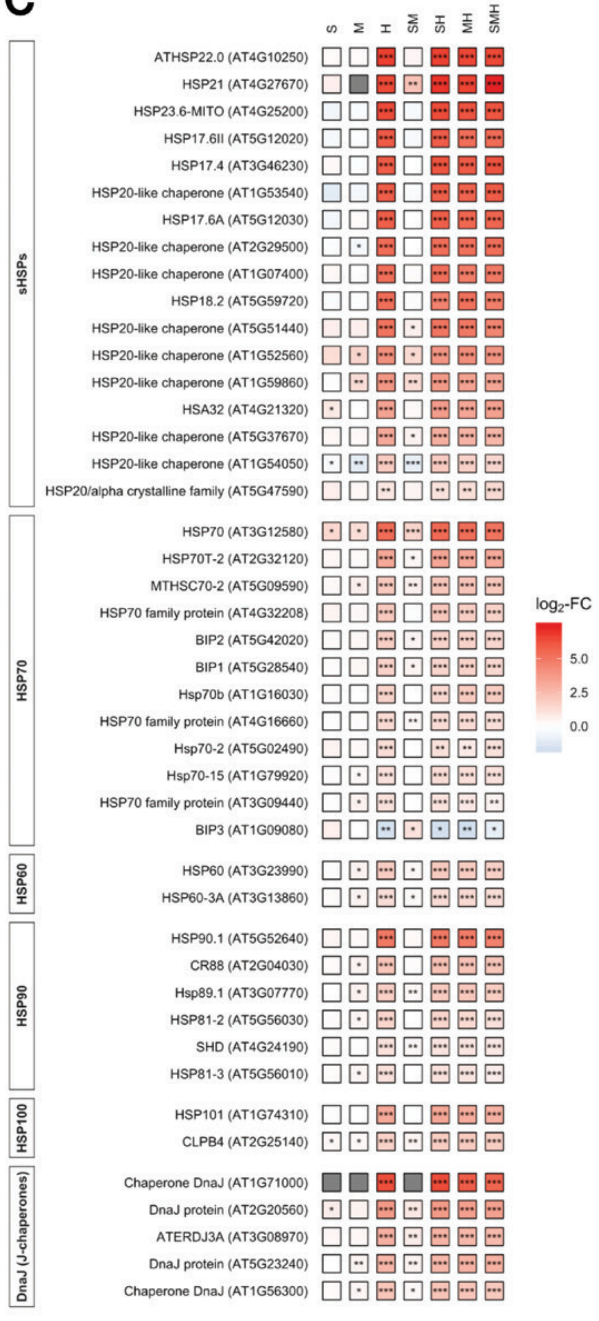

\section{B}

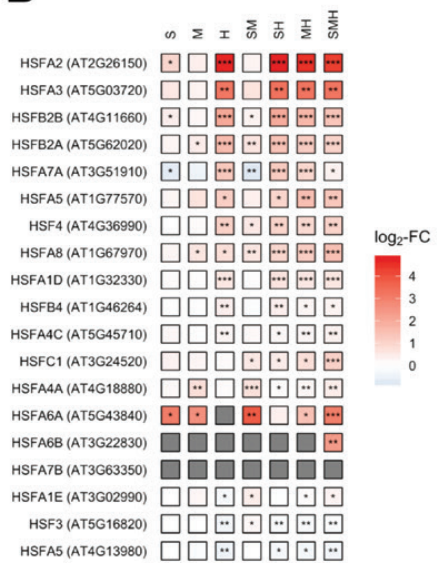

Fig. 4. Changes in gene expression under individual and combined stress treatments of top 50 genes of cluster 1 showing antagonistic effects between osmotic and heat treatments (A), HSFs (B) and HSPs (C). Shown are the log2-FC values compared with control under the different treatments in false color code from enhanced (red) to repressed (blue) expression. Asterisks indicate differential abundance between respective treatment and control as determined by sleuth $\left({ }^{\star} q<0.05 ;{ }^{* *} q<0.01 ;{ }^{* \star} q<0.001\right)$. H, heat; M, mannitol; S, salt.

environmental stress causing serious damage to cellular proteins. Notably, BIP3, an endoplasmic reticulum HSP70, was the only HSP gene repressed by heat. Instead, we found that BIP3 was induced by salt stress (Fig. 4C); BIP3's involvement in the unfolding protein response caused by salinity was recently discussed (Henriquez-Valencia et al., 2015; Guan et al., 2018). 
Abiotic stress combinations induce intensive reprograming of the expression profiles of various transcription factor families

In all living organisms, transcription factors (TFs) represent the master control components that dynamically alter the cellular transcriptome, which in turn adapts metabolism and phenotype to the surrounding environment (Riaño-Pachón et al., 2007; Mitsuda and Ohme-Takagi, 2009). Here, we investigated the interacting effects of various stresses on the expression profiles of the $1187 \mathrm{TF}$ genes annotated in the Arabidopsis genome (see Supplementary Table S3). As genes encoding TFs frequently do not show high expression changes compared with non-regulatory genes (Cheng et al., 2007), we monitored the changes in the expression profile of the TFs at a cut-off value of 1.5-FC. With this threshold we found that $701 \mathrm{TF}$ genes were significantly differentially expressed by at least one stress treatment compared with the control (Supplementary Table S3).

The AP2-EREBP, WRKY, bHLH, and MYP/MYP related TF families showed the highest responsiveness to the applied stress combinations. We found that the members of a given TF family did not exhibit similar expression patterns under a given stress condition (see Supplementary Table S3). As a case study, we analysed the changes in the expression patterns of the gene members of the WRKY TF family. These TFs are assembled into three main groups (I, II, and III) based on the number of WRKY domains and the features of their zinc-finger-like motif (Eulgem et al., 2000; Rushton et al., 2010). In our study, 29 out of 72 genes annotated as WRKY TFs were differential expressed by at least one of the seven stress treatments compared with control (Fig. 5A). After categorizing the genes into induced or repressed sets, we found that there is no specific expression trend among the members of the same groups or even subgroups of this TF family under the applied stress treatments (Fig. 5A). This analysis indicates that the categorization of TF genes based on their binding motifs does not correlate with their expected biological functions inferred from their responsiveness to different environmental conditions. This raises the historic question related to genome-wide studies, of whether proteins that are identified according to their sequence similarities with known proteins are actually correctly categorized (Riechmann, 2002).

\section{Genes involved in cell cycle are down-regulated especially under heat stress}

Various events of the cell cycle in eukaryotes are controlled by cyclin-dependent kinases and their associated activators, cyclins, and cell division cycle proteins (Komaki and Sugimoto, 2012). Our data showed that the expression of many genes coding for these proteins was reduced mainly by the stress treatments that included heat, and particularly under the triple stress treatment
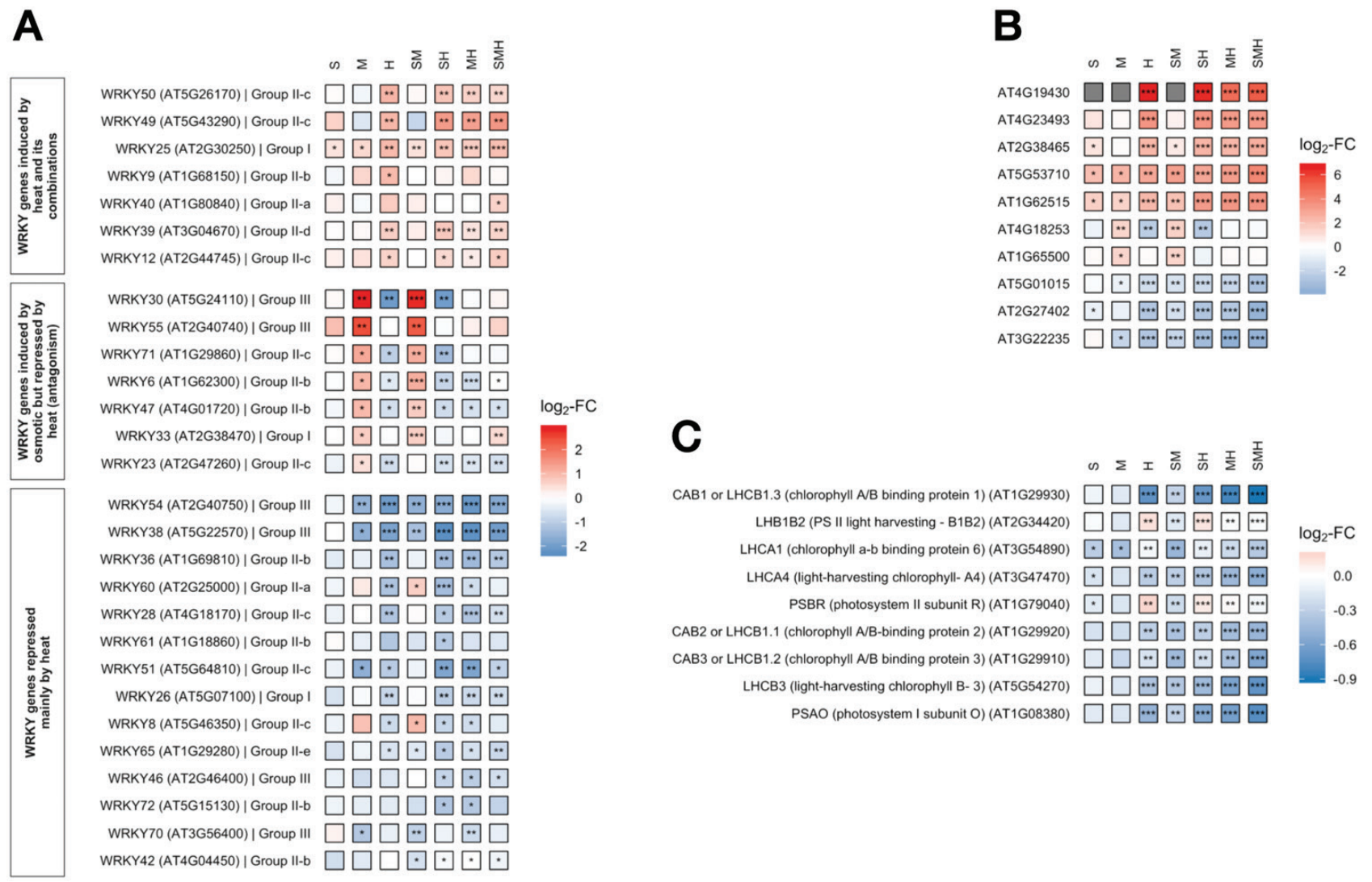

Fig. 5. Changes in gene expression under individual and combined stress treatments of gene members of WRKY TF family (A), 10 selected genes of unknown functions (B) and abundant photosynthetic genes (C). Shown are the log2-FC values compared with control under the different treatments in false color code from enhanced (red) to repressed (blue) expression. Asterisks indicate differential abundance between respective treatment and control as determined by sleuth $\left({ }^{*} q<0.05 ;{ }^{* \star} q<0.01 ;{ }^{* *} q<0.001\right)$. H, heat; M, mannitol; S, salt. 
(see Supplementary Table S4). Furthermore, we found that many genes involved in DNA synthesis and repair were also down-regulated mainly by heat and combinations of stresses including heat, and many genes involved in cell division were specifically repressed by heat (Supplementary Table S4).

\section{Almost $40 \%$ of the differentially expressed genes under stress treatments are of unknown function}

In the Arabidopsis genome, 38\% (10 415 genes) of the total number of genes are of unknown function. Here, we found that out of these, 1312 genes are significantly $(P<0.05)$ differentially expressed $\geq 2-\mathrm{FC}$ by at least one of the seven stress treatments compared with control (see Supplementary Table S5). Interestingly, this number represents $37 \%$ of the total number (3529) of the differentially expressed genes.

Ten genes attracted our attention as they show specific expression patterns under various stress treatments (Fig. 5B).Three genes, AT4G19430, AT4G23493, and AT2G38465, were specifically highly induced by heat, suggesting that they may play an important role in thermotolerance. Two genes, AT5G53710 and AT1G62515, showed high induction by all individual stress treatments. Two genes, AT4G18253 and AT1G65500, were specifically induced by osmotic stress. Finally, AT5G01015, AT2G27402, and AT3G22235, showed significant repression by all treatments, with very strong repression under the combined triple stress treatment.

\section{The transcription of most abundant genes is down- regulated under stress}

Abundantly expressed genes are usually ignored or underestimated in analyses that only use FC to evaluate changes in gene expression. The analysis of genes with transcript abundances higher than 100 TPM, regardless their FC expression levels (see Supplementary Table S6 ), indicated that around $70 \%$ of these genes were repressed by the stress treatments. This observation most probably reflects a strategy of the stressed plants to save resources for other urgent tasks required for defense and survival. Out of the considered 1397 abundantly expressed genes, 336 genes were differentially expressed by salinity (out of them 289 genes were repressed), 296 genes were differentially expressed by osmotic stress (out of them 226 were repressed), 1173 genes were differentially expressed by treatments that included heat (out of them 731 were repressed), and 1332 were differentially expressed by the triple stress treatment (out of them 962 were repressed) (Supplementary Table S6).

When we only considered the relative FC in expression levels, we inferred that the salt treatment has a negligible effect on expression patterns of Arabidopsis genome (see Supplementary Table S6). Nevertheless, by considering the TPM we found a hidden effect of salinity on the expression patterns of abundantly expressed genes. Among the salinity-responsive genes, photosynthetic genes were the most significantly repressed (Supplementary Table S6; Fig. 5C).
The expression of the most abundant photosynthetic genes is highly down-regulated under stress

Out of the considered abundant genes, around 100 genes are involved in photosynthesis (see Supplementary Table S6). If only the relative FC expression levels are considered, 90 of those photosynthetic genes would be disregarded (Supplementary Table S6). The changes in expression levels of these genes are actually significant, with a general trend of down-regulation. Analysis of the top 10 most abundant photosynthetic genes (Fig. 5C) showed that most of these genes are repressed by heat and an intensification of this response is observed when other stresses simultaneously act with heat.

The most abundant photosynthetic genes encode the subunits of ribulose bisphosphate carboxylase/oxygenase (Rubisco) and the light harvesting complexes (LHC) (see Supplementary Table S6). Indeed, on Earth, Rubisco is the most abundant soluble protein (Ellis, 1979; Raven, 2013; Bar-On and Milo, 2019), and light harvesting $a / b$ binding proteins are suggested to be the most abundant membrane-bound proteins (Xu et al., 2012). The expression level of the Rubisco gene $R B C S-1 A$ was repressed by the seven stress treatments, with the highest repression occurring under the combined triple treatment (Fig. 5C). In accordance with the reduction in the transcriptional expression of Rubisco, its activity was also found to be inhibited under drought and heat stresses in different plant species (Fahad et al., 2017; Haworth et al., 2018). We also found that LHCII genes are also repressed by stress treatments, particularly by heat and its combinations (Fig. 5C). In addition, our results showed that two LCHII genes, OHP1 and $\mathrm{OHP2}$, are abundant and repressed by the stress combinations (Supplementary Table S6). Arabidopsis mutant lines of both genes, ohp 1 and ohp 2 , showed severe reduction in growth, decrease in pigmentation and abnormal thylakoid architecture (Hey and Grimm, 2018; Li et al., 2019). Probably, the downregulation of these important genes explains, at least partially, the plant growth reduction observed under abiotic stresses (Fig. 1B).

\section{Treatments that include heat stress cause down- regulation of most ribosomal genes}

Among the abundantly expressed genes are those involved in the synthesis of ribosomal proteins. We found that 234 abundant genes coding for ribosomal proteins were significantly repressed by treatments involving heat, especially by the triple stress treatment (see Supplementary Table S6). Most of these genes are predicted to localize to the cytosol (Supplementary Table S6), where the bulk of the protein synthesis occurs. It was reported that stress does not dramatically modify the basal expression patterns of most genes encoding cytoplasmic ribosomal protein (Sormani et al., 2011). However, considering the high expression levels of the ribosomal genes, small changes in the relative expression levels represent big changes in protein amounts, which most probably is transduced in significant functional effects. This observed down-regulation of expression of the abundant ribosomal proteins parallels the downregulation of expression of the most abundant proteins in plant cells, Rubisco and LHC (Supplementary Table S6; Fig. 5C). 
The profile of specific metabolites is affected by the abiotic stress treatments

We further investigated the effects of the different stress treatments on the profiles of several metabolites in leaves of Arabidopsis using GC-MS (see Supplementary Table S7). A PCA revealed that the PC1 separated the samples of the treatments that include heat stress from all other treatments, explaining $42.8 \%$ of the total variance within the dataset (Fig. 6A). Other components of the analysis did not provide any further clear difference between the treatments.

Our results revealed that specific amino acids were coordinately affected by treatments that included heat stress. Glycine, alanine, valine, isoleucine, lysine, tyrosine, and phenylalanine clustered together in a Pearson correlation heatmap and show increased levels under treatments with a heat stress component (Figs 6B, 7). We also observed that the levels of the osmoprotective amino acid proline were specifically higher following salt treatment during single or combined application with other stresses (Fig. 7). Treatment with mannitol also enhanced the levels of proline but with less intensity (Fig. 7). Accumulation of proline was also recorded in different plant species under salinity and drought stresses (Szabados and Savouré, 2010).

Soluble sugars are highly sensitive to environmental stresses, as they represent the supply of carbohydrates from source to sink organs and are also involved in osmoprotection. Our results showed that raffinose, galactinol, and trehalose, which all have osmoprotective properties, were highly increased by all treatments that included heat stress, while other treatments had a positive but much lower influence (Figs 6B, 7). Our

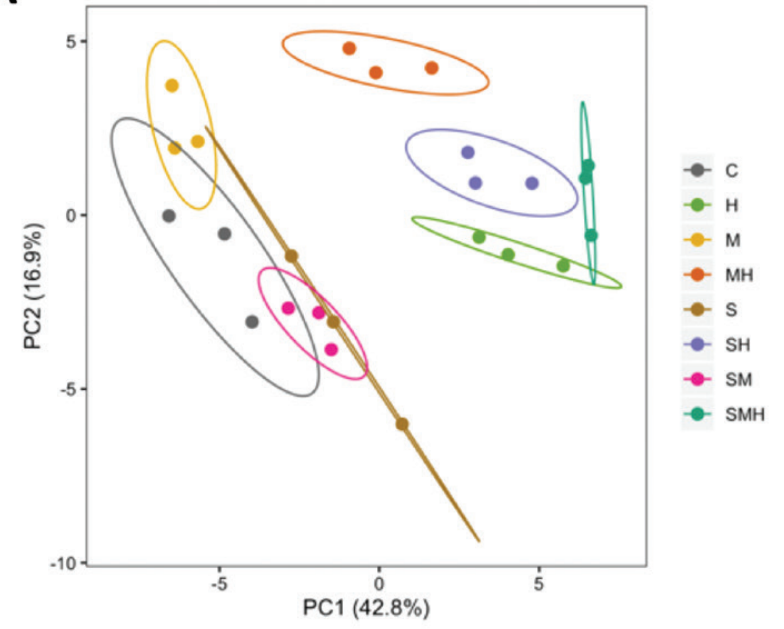

B

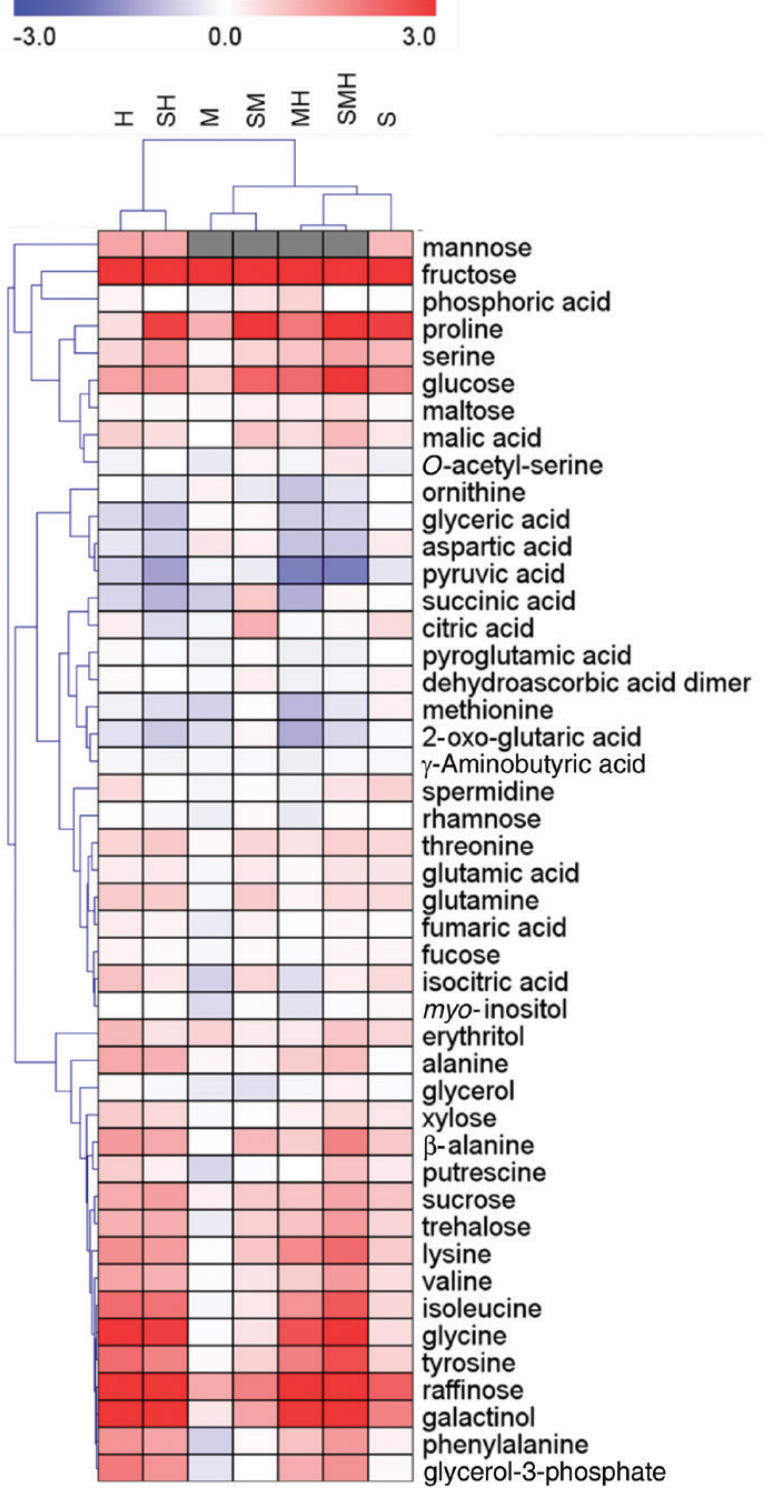

Fig. 6. (A) Principal component analysis of relative metabolite level data. (B) Hierarchical clustering heatmap representation of changes in relative metabolite levels measured in leaves of Arabidopsis after stress treatments. Hierarchical clustering was applied to rows and columns using Pearson's correlation coefficient. Red or blue indicate that the metabolite content is increased or decreased compared the control, respectively. Values presented are means of three replicates and are shown in false-color code. C, control; $\mathrm{H}$, heat; $\mathrm{M}$, mannitol; S, salt. 


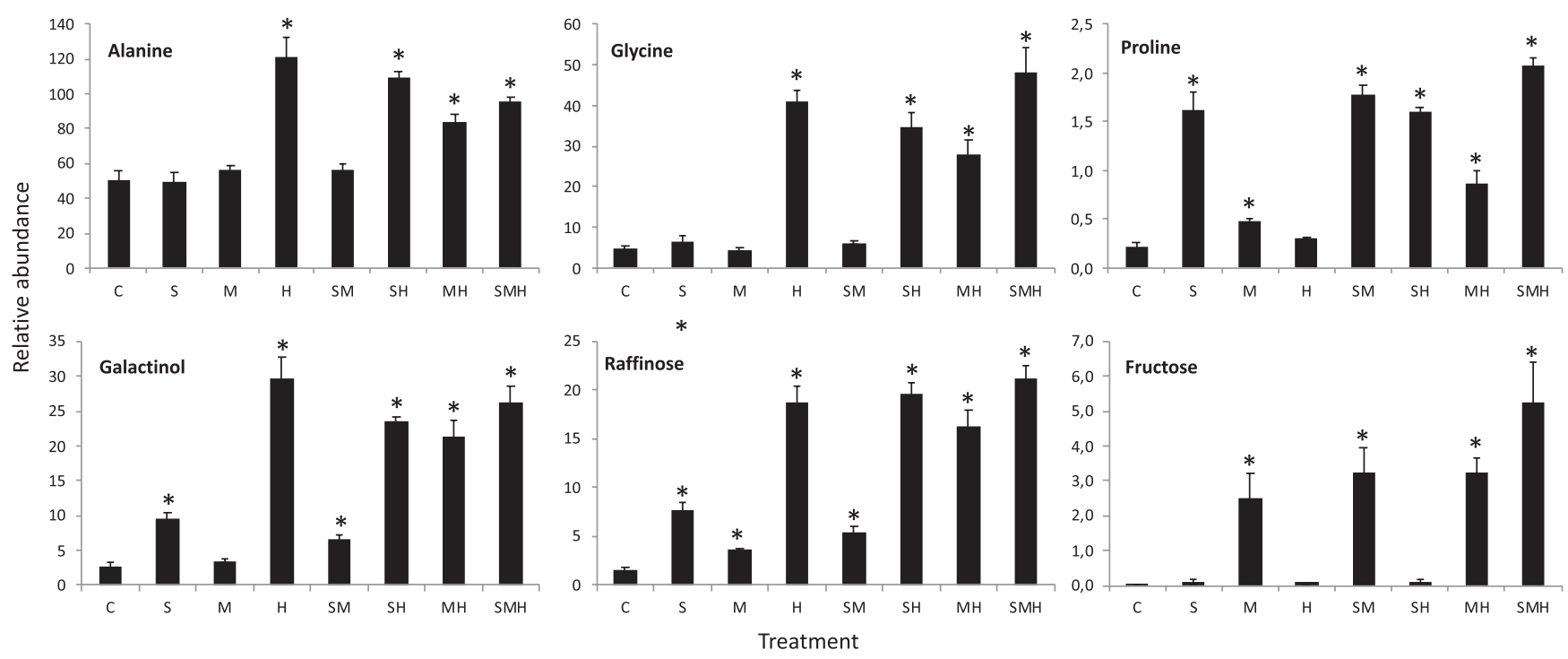

Fig. 7. Relative abundances of selected metabolites to the internal control as monitored by GC-MS under different stress treatments. Values presented are the mean of three biological replicates \pm SE. Asterisks indicate significant differences from the control as assessed by Student's $t$-test. ${ }^{*} P \leq 0.05$. C, control; H, heat; M, mannitol; S, salt.

transcriptomic data indicate that the expression of three genes encoding enzymes involved in the biosynthesis of galactinol and raffinose (Go1S1,-2, and -3) are enhanced under the imposed stress treatments (see Supplementary Table S1, cluster 6). In line with these results, transgenic Arabidopsis overexpressing Gols2 have enhanced levels of galactinol and raffinose and are more tolerant to drought and salinity stresses (Taji et al., 2002). Galactinol and raffinose also protect plants from oxidative damage (Nishizawa et al., 2008). We also found significant increment in the levels of fructose, a free sugar with osmoprotective function, specifically under treatments that include osmotic stress (Fig. 7).

\section{Discussion}

\section{Heat has a dominant effect on global gene expression}

This study focused on molecular plant responses to combined global warming-associated abiotic stresses. The data presented here provide evidence that high temperature strongly affects global gene expression and metabolite profiles and governs the plant response even in combination with salinity and osmotic stresses.

The observed dominant effects of heat on the expression of a large number of genes (Figs 2,3) can be mainly ascribed to the hypersensitivity of plant membranes to high temperature, which rapidly affects the structures and functions of biological membranes (Niu and Xiang, 2018). As most of the biological functions of the plants require molecular interactions through cellular and organellar membranes (Suzuki et al., 2012; Pospíšil and Prasad, 2014), the plant seems to quickly reorganize its transcriptome to rapidly and efficiently cope with the deleterious effects of heat stress. In addition to this, high temperature is mostly responsible for protein misfolding and enhanced protein degradation (McLoughlin et al., 2019). In line with this, we found that most members of all HSP families are specifically induced by treatments that include heat stress, indicating that high temperature is the most severe environmental stress causing serious damage to cellular proteins. Longer heat waves accompanying the global climate changes are expected to dramatically exacerbate these effects (IPCC, 2014; Haworth et al., 2018).

We also found that the mitochondrial activity is highly responsive to temperature stress (Supplementary Table S1). The increased abundance of the mitochondrial transcripts observed could represent an up-regulation of gene expression to replace damaged and degraded proteins. In accordance with this, a proteome analysis of cauliflower mitochondria indicated that the abundance of some oxidative phosphorylation components specifically increases in response to high temperature (Rurek et al., 2018). Changes in complex I composition most probably influence the redox homeostasis of mitochondria, further impacting the expression of antioxidant defense components and stress-responsive genes in a retrograde fashion. Furthermore, we found that genes involved in controlling cell cycle, cell division, DNA synthesis, and DNA repair are downregulated especially under heat stress (Supplementary Table S4). These observations suggest that under high temperatures, plants tend to arrest their cell cycles. While this response can lead to reduced yield in crops, it has an important adaptive significance as a survival strategy (Kitsios and Doonan 2011).

\section{Abundant and unknown genes in the spotlight}

In our study, we draw attention to two categories of genes that are usually disregarded in genome-wide studies: those annotated as of unknown function and those having high transcript levels under normal growth conditions.

\section{As a tradeoff strategy, plants down-regulate the transcription of most abundant genes under abiotic stress}

Our analysis indicated that abundantly expressed genes, such as those encoding photosynthetic and ribosomal proteins, 
are down-regulated specifically in response to heat stress (Supplementary Table S6; Fig. 5C); an intensification of this response was observed when other stresses acted simultaneously with heat. These responses most probably reflect a strategy of the plants to save resources and energy that can instead be invested into defense mechanisms. Maintaining an efficient use of limited resources, such as nitrogen, through the reduction of expression of photosynthetic proteins represents an acclimation strategy to changing environmental factors (Pao et al., 2019). The reduction in photosynthesis and growth rates of plants observed in many studies (Fahad et al., 2017; Haworth et al., 2018; Huang et al., 2019) may be, at least partly, due to this plant strategy, and may not only be a direct consequence of the damaging effects of the applied stresses. However, it cannot be ruled out that the decreased expression of abundant transcripts for photosynthetic proteins could result from feedback control of these genes, as carbon fixation is inhibited by closed stomata and a reduced demand for photosynthates due to reduced growth.

In addition to the down-regulation of genes coding for photosynthetic proteins, our results indicate that the expression of a number of genes involved in the degradation of chloroplastic proteins, such as FtsH6, FtsH8, and FtsH12, was up-regulated by heat and its stress combinations (see Supplementary Table S8). FtsH6 was reported to be involved in degradation of the PSII light harvesting proteins Lhcb1 and Lhcb3 under senescence and high-light conditions (Zelisko et al., 2005; Sedaghatmehr et al., 2016). Indeed, highly regulated degradation of the LHCII represents a crucial action to avoid photochemical damage to chloroplasts and other cellular constituents under harsh conditions (Zelisko et al., 2005). This adaptive down-regulation of the photosynthetic machinery, especially under prolonged heat waves, will inevitably inhibit the overall plant performance, diminishing plant growth.

Some functionally uncharacterized genes are candidates for engineering plant abiotic stress tolerance

Interestingly, $38 \%$ of the Arabidopsis genome is of unknown function, and $37 \%$ of the genes that were significantly affected by stress treatments were also of unknown function (Supplementary Table 5). The expression of a group of these genes of unknown functions was highly modified under specific treatments applied (Fig. 5B). This highlights them as candidate genes for functional characterization and for breeding programs seeking to increase stress tolerance in crops.

\section{Different compounds act as compatible solutes depending on the abiotic stress applied}

Our metabolite analysis indicated the accumulation of specific compatible solutes depending on the nature of the stress applied (Figs 6B, 7). This most probably reflects the differential activation of specific metabolic pathways and the necessity to avoid cellular toxic effects of given osmoprotectants.
These results therefore highlight the importance of analysing stress-associated metabolites under specific stresses in programs aimed at engineering stress tolerance.

We also found a coordinated increase in the levels of some free amino acids under heat stress (Fig. 6B), which most probably reflects the catabolism of proteins to ensure the survival of the whole organism at the expense of other cellular processes. In line with this scenario, catabolic pathways for several amino acids were found to be induced under abiotic stress conditions, suggesting their use as a respiratory substitute to compensate the down-regulation of photosynthesis (Araújo et al., 2011; Batista-Silva et al., 2019). It is also possible that the enhanced levels of the branched-chain and aromatic amino acids reflect an increased production of secondary metabolites during the stress responses (Vetter, 2000; Dixon, 2001). Nevertheless, high temperatures can also negatively impact secondary metabolite production. This is illustrated by the case of fruits of Vitis vinifera and Ribes nigrum, in which reduced downstream polyphenol metabolism at high temperatures results in increased levels of phenylalanine (Mori et al., 2007; Allwood et al, 2019).

\section{Concluding remarks}

The general down-regulation of highly transcribed genes and cell cycle genes, combined with increased protein degradation, suggests that under unfavorable environmental conditions, Arabidopsis enters an emergency state with arrested growth and enhanced activity of molecular mechanisms for survival, causing a major reduction of plant development and productivity (Fig. 8). It can be argued that these responses are particularly important for a wild plant in a natural ecosystem, where the effects of environmental stresses are likely to be more dramatic than for a crop plant. Nevertheless, it is well documented that environmental stresses also significantly reduce crop productivity (Bita and Gerats, 2013; Lesk et al., 2016). Our findings provide molecular evidence for the serious effects of global warming on world food security, and the necessity of developing strategies to sustain future crop productivity.

\section{Supplementary data}

Supplementary data are available at JXB online.

Dataset S1. Quantitative information and annotation for all reads mapped onto the reference genome of Arabidopsis.

Table S1. List of all differentially expressed genes (3529 genes) at 2-FC analysed by $k$-means clustering.

Table S2. Expression of Arabidopsis HSP genes (156 genes) under seven stress treatments compared with control.

Table S3. Expression of all Arabidopsis TF genes (1187 genes) under seven stress treatments compared with control. 


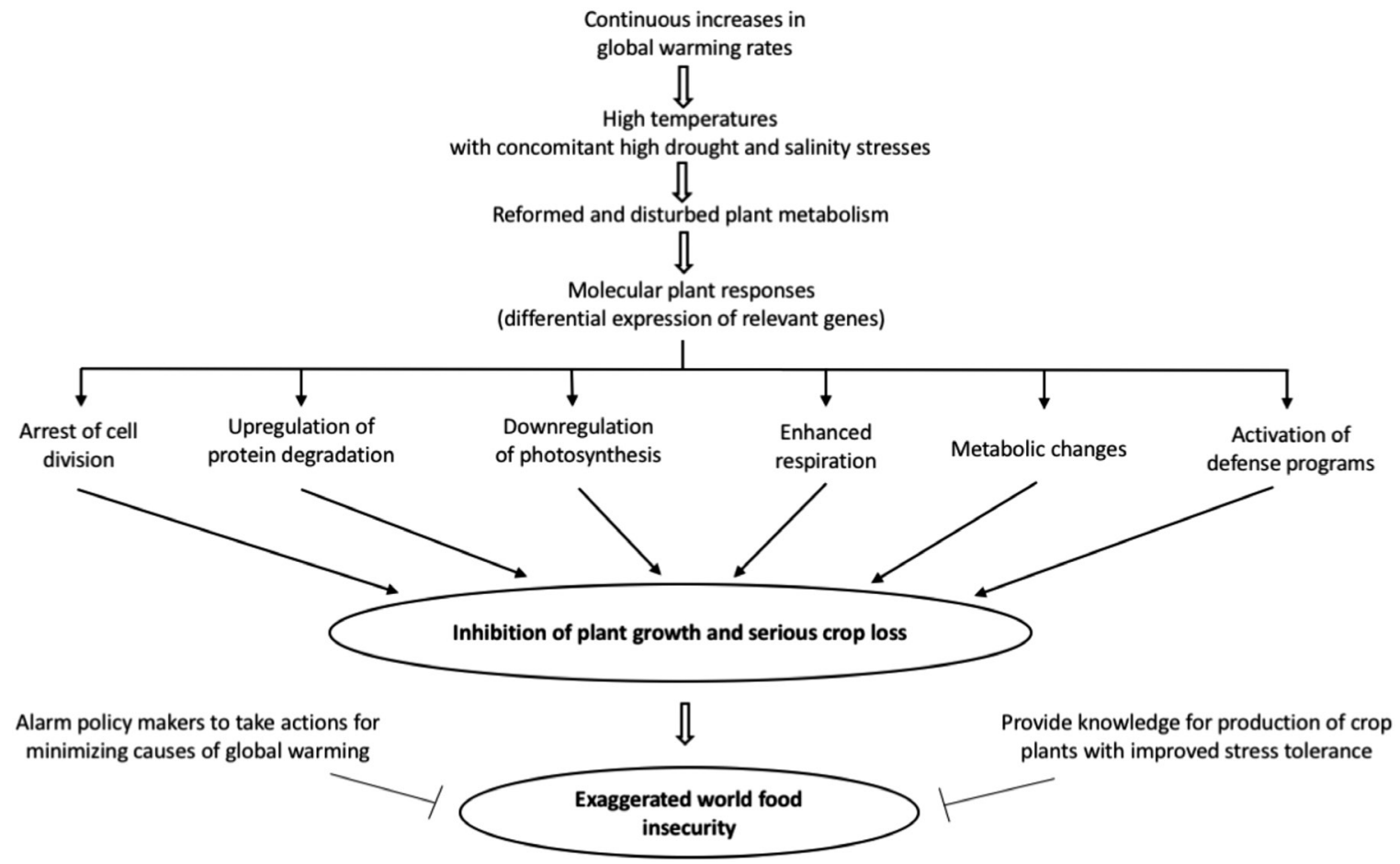

Fig. 8. A schematic representation of molecular plant responses to combined global warming-associated abiotic stresses that lead to reduction of crop yield as depicted from the presented and discussed results in the current study.

Table S4. Expression of Arabidopsis genes involved in cell division and DNA repair/synthesis at 2-FC under seven stress treatments compared with control.

Table S5. Expression of 1312 genes annotated as having unknown function, differentially expressed by at least one treatment.

Table S6. Expression of 1397 genes with transcript abundances higher than 100 transcripts per million.

Table S7. Profiles of several metabolites in leaves of Arabidopsis using GC-MS.

Table S8. Expression of genes involved in protein degradation with $>2$-FC.

\section{Acknowledgements}

Financial support was provided by the Deutsche Forschungsgemeinschaft (DFG, German Research Foundation) through grant MA2379/11-2 to VGM and Germany's Excellence Strategy-EXC-2048/1— project ID 390686111. Work of NS was in part funded by the Ministry of Higher Education of the Arab Republic of Egypt (MoHE), the Deutscher Akademischer Austauschdienst (DAAD) and a StayConnected-Grant of the Heinrich Heine University Düsseldorf.ARF and SA acknowledge the PlantaSYST project by the EU Horizon 2020 Research and Innovation Programme (SGA-CSA 664621 and 739582 under FPA 664620). We acknowledge the technical assistance of Samantha Flachbart for preparation of Illumina libraries.

\section{Data availability}

The read data were deposited at the National Center for Biotechnology Information Gene Expression Omnibus under accession number
GSE146206 cgi?acc $=$ GSE146206).

\section{References}

Allwood JW, Woznicki TL, Xu Y, et al. 2019. Application of HPLCPDA-MS metabolite profiling to investigate the effect of growth temperature and day length on blackcurrant fruit. Metabolomics 15, 12.

Araújo WL, Tohge T, Ishizaki K, Leaver CJ, Fernie AR. 2011. Protein degradation - an alternative respiratory substrate for stressed plants. Trends in Plant Science 16, 489-498.

Atkinson NJ, Urwin PE. 2012. The interaction of plant biotic and abiotic stresses: from genes to the field. Journal of Experimental Botany $\mathbf{6 3}$ 3523-3543.

Barah P, Naika M, Jayavelu ND, Sowdhamini R, Shameer K, Bones AM. 2016. Transcriptional regulatory networks in Arabidopsis thaliana during single and combined stresses. Nucleic Acids Research 44, 3147-3164.

Bar-On YM, Milo R. 2019. The global mass and average rate of rubisco. Proceedings of the National Academy of Sciences, USA 116, 4738-4743.

Batista-Silva W, Heinemann B, Rugen N, Nunes-Nesi A, Araújo WL, Braun HP, Hildebrandt TM. 2019. The role of amino acid metabolism during abiotic stress release. Plant, Cell \& Environment 42, 1630-1644.

Battisti DS, Naylor RL. 2009. Historical warnings of future food insecurity with unprecedented seasonal heat. Science 323, 240-244.

Benjamini Y, Hochberg Y. 1995. Controlling the false discovery rate: a practical and powerful approach to multiple testing. Journal of the Royal Statistical Society 57, 289-300.

Bita CE, Gerats T. 2013. Plant tolerance to high temperature in a changing environment: scientific fundamentals and production of heat stress-tolerant crops. Frontiers in Plant Science 4, 273.

Bray NL, Pimentel H, Melsted P, Pachter L. 2016. Near-optimal probabilistic RNA-seq quantification. Nature Biotechnology 34, 525-527.

Cheng C, Yan X, Sun F, Li LM. 2007. Inferring activity changes of transcription factors by binding association with sorted expression profiles. BMC Bioinformatics 8, 452. 
Deryng D, Conway D, Ramankutty N, Price J, Warren R. 2014. Global crop yield response to extreme heat stress under multiple climate change futures. Environmental Research Letters 9, 034011.

Dixon RA. 2001. Natural products and plant disease resistance. Nature 411, 843-847.

Ellis RJ. 1979. The most abundant protein in the world. Trends in Biochemical Sciences 4, 241-244.

Eulgem T, Rushton PJ, Robatzek S, Somssich IE. 2000. The WRKY superfamily of plant transcription factors. Trends in Plant Science $\mathbf{5}$, 199-206.

Fahad S, Bajwa AA, Nazir U, et al. 2017. Crop production under drought and heat stress: plant responses and management options. Frontiers in Plant Science 8, 1147.

Georgii E, Jin M, Zhao J, Kanawati B, Schmitt-Kopplin P, Albert A, Winkler JB, Schäffner AR. 2017. Relationships between drought, heat and air humidity responses revealed by transcriptome-metabolome co-analysis. BMC Plant Biology 17, 120.

Goyal K, Walton LJ, Tunnacliffe A. 2005. LEA proteins prevent protein aggregation due to water stress. The Biochemical Journal $\mathbf{3 8 8}$, 151-157.

Guan P, Wang J, Li H, Xie C, Zhang S, Wu C, Yang G, Yan K, Huang J, Zheng C. 2018. SENSITIVE TO SALT1, an endoplasmic reticulum-localized chaperone, positively regulates salt resistance. Plant Physiology 178, 1390-1405.

Haworth M, Marino G, Brunetti C, Killi D, De Carlo A, Centritto M. 2018. The impact of heat stress and water deficit on the photosynthetic and stomatal physiology of olive (Olea europaea L.) - A case study of the 2017 heat wave. Plants 7, E76.

Heath RL, Packer L. 1968. Photoperoxidation in isolated chloroplasts. I. Kinetics and stoichiometry of fatty acid peroxidation. Archives of Biochemistry and Biophysics 125, 189-198.

Henriquez-Valencia C, Moreno AA, Sandoval-lbañez O, Mitina I, Blanco-Herrera F, Cifuentes-Esquivel N, Orellana A. 2015. bZIP17 and bZIP60 regulate the expression of BiP3 and other salt stress responsive genes in an UPR-independent manner in Arabidopsis thaliana. Journal of cellular biochemistry 116, 1638-1645.

Hey D, Grimm B. 2018. ONE-HELIX PROTEIN2 (OHP2) is required for the stability of OHP1 and assembly factor HCF244 and is functionally linked to PSIl Biogenesis. Plant Physiology 177, 1453-1472.

Huang J, Minnis P, Yan H, Yi Y, Chen B, Zhang L, Ayers JK. 2010. Dust aerosol effect on semi-arid climate over Northwest China detected from A-Train satellite measurements. Atmospheric Chemistry and Physics 10, 6863-6872.

Huang R, Liu Z, Xing M, Yang Y, Wu X, Liu H, Liang W. 2019. Heat stress suppresses Brassica napus seed oil accumulation by inhibition of photosynthesis and BnWRI1 pathway. Plant \& Cell Physiology 60, 1457-1470.

IPCC. 2014. Climate Change 2014: Impacts, adaptation, and vulnerability. Working Group II Contribution of to the Fifth Assessment Report of the Intergovernmental Panel on Climate Change. Cambridge, UK: Cambridge University Press.

IPCC. 2018. Summary for policymakers. In: Masson-Delmotte V, Zhai P, Pörtner $\mathrm{HO}$, et al. eds. Global warming of $1.5^{\circ} \mathrm{C}$. An IPCC Special Report on the impacts of global warming of $1.5^{\circ} \mathrm{C}$ above pre-industrial levels and related global greenhouse gas emission pathways, in the context of strengthening the global response to the threat of climate change, sustainable development, and efforts to eradicate poverty. Geneva, Switzerland: World Meteorological Organization.

Kilian J, Whitehead D, Horak J, Wanke D, Weinl S, Batistic O, D'Angelo C, Bornberg-Bauer E, Kudla J, Harter K. 2007. The AtGenExpress global stress expression data set: protocols, evaluation and model data analysis of UV-B light, drought and cold stress responses. The Plant Journal 50, 347-363.

Kitsios G, Doonan JH. 2011. Cyclin dependent protein kinases and stress responses in plants. Plant Signaling \& Behavior 6, 204-209.

Komaki S, Sugimoto K. 2012. Control of the plant cell cycle by developmental and environmental cues. Plant \& Cell Physiology 53, 953-964.

Lawlor DW. 2013. Genetic engineering to improve plant performance under drought: physiological evaluation of achievements, limitations, and possibilities. Journal of Experimental Botany 64, 83-108.
Lee D, Polisensky DH, Braam J. 2005. Genome-wide identification of touch- and darkness-regulated Arabidopsis genes: a focus on calmodulinlike and XTH genes. New Phytologist 165, 429-444.

Lesk C, Rowhani P, Ramankutty N. 2016. Influence of extreme weather disasters on global crop production. Nature 529, 84-87.

Li Y, Liu B, Zhang J, Kong F, Zhang L, Meng H, Li W, Rochaix JD, Li D, Peng L. 2019. OHP1, OHP2, and HCF244 form a transient functional complex with the photosystem II reaction center. Plant Physiology 179, 195-208.

Lisec J, Schauer N, Kopka J, Willmitzer L, Fernie AR. 2006. Gas chromatography mass spectrometry-based metabolite profiling in plants. Nature Protocols 1, 387-396.

Lobell DB, Field CB. 2007. Global scale climate-crop yield relationships and the impacts of recent warming. Environmental Research Letters 2, 004000.

Lobell DB, Schlenker W, Costa-Roberts J. 2011. Climate trends and global crop production since 1980. Science 333, 616-620.

Luedemann A, Strassburg K, Erban A, Kopka J 2008. TagFinder for the quantitative analysis of gas chromatography-mass spectrometry (GC-MS)based metabolite profiling experiments. Bioinformatics 24, 732-737.

Matsui A, Ishida J, Morosawa T, et al. 2008. Arabidopsis transcriptome analysis under drought, cold, high-salinity and ABA treatment conditions using a tiling array. Plant \& Cell Physiology 49, 1135-1149.

McLoughlin F, Kim M, Marshall RS, Vierstra RD, Vierling E. 2019. HSP101 interacts with the proteasome and promotes the clearance of ubiquitylated protein aggregates. Plant Physiology 180, 1829-1847.

Mitsuda N, Ohme-Takagi M. 2009. Functional analysis of transcription factors in Arabidopsis. Plant \& Cell Physiology 50, 1232-1248.

Mittler R. 2006. Abiotic stress, the field environment and stress combination. Trends in Plant Science 11, 15-19.

Mori K, Goto-Yamamoto N, Kitayama M, Hashizume K. 2007. Effect of high temperature on anthocyanin composition and transcription of flavonoid hydroxylase genes in 'Pinot noir' grapes (Vitis vinifera). The Journal of Horticultural Science and Biotechnology 82, 199-206.

Munns R, Tester M. 2008. Mechanisms of salinity tolerance. Annual Review of Plant Biology 59, 651-681.

Nishizawa A, Yabuta Y, Shigeoka S. 2008. Galactinol and raffinose constitute a novel function to protect plants from oxidative damage. Plant Physiology 147, 1251-1263.

Niu Y, Xiang Y. 2018. An overview of biomembrane functions in plant responses to high-temperature stress. Frontiers in Plant Science $\mathbf{9}, 915$.

Osthoff A, Donà Dalle Rose P, Baldauf JA, Piepho HP, Hochholdinger F. 2019. Transcriptomic reprogramming of barley seminal roots by combined water deficit and salt stress. BMC Genomics 20, 325.

Pandey P, Ramegowda V, Senthil-Kumar M. 2015. Shared and unique responses of plants to multiple individual stresses and stress combinations: physiological and molecular mechanisms. Frontiers in Plant Science 6, 723. Pao YC, Chen TW, Moualeu-Ngangue DP, Stützel H. 2019. Environmental triggers for photosynthetic protein turnover determine the optimal nitrogen distribution and partitioning in the canopy. Journal of Experimental Botany 70, 2419-2434.

Park C-E, Jeong S-J, Joshi M, et al. 2018. Keeping global warming within $1.5^{\circ} \mathrm{C}$ constrains emergence of aridification. Nature Climate Change 8, 70-74.

Pimentel H, Bray NL, Puente S, Melsted P, Pachter L. 2017. Differential analysis of RNA-seq incorporating quantification uncertainty. Nature Methods 14, 687-690.

Pospíšil P, Prasad A. 2014. Formation of singlet oxygen and protection against its oxidative damage in Photosystem II under abiotic stress. Journal of Photochemistry and Photobiology. B, Biology 137, 39-48.

Prasch CM, Sonnewald U. 2013. Simultaneous application of heat, drought, and virus to Arabidopsis plants reveals significant shifts in signaling networks. Plant Physiology 162, 1849-1866.

Prasch CM, Sonnewald U. 2015. Signaling events in plants: stress factors in combination change the picture. Environmental and Experimental Botany 114, 4-14.

R Core Team. 2018. R: A language and environment for statistical computing. Vienna, Austria: R Foundation for Statistical Computing. 
Rasmussen S, Barah P, Suarez-Rodriguez MC, Bressendorff S, Friis P, Costantino P, Bones AM, Nielsen HB, Mundy J. 2013. Transcriptome responses to combinations of stresses in Arabidopsis. Plant Physiology 161, 1783-1794.

Raven JA. 2013. Rubisco: still the most abundant protein of Earth? New Phytologist 198, 1-3.

Riaño-Pachón DM, Ruzicic S, Dreyer I, Mueller-Roeber B. 2007. PInTFDB: an integrative plant transcription factor database. BMC Bioinformatics 8, 42.

Riechmann JL. 2002. Transcriptional regulation: a genomic overview. The Arabidopsis Book 1, e0085.

Ritossa F. 1962. A new puffing pattern induced by temperature shock and DNP in Drosophila. Experientia 18, 571-573.

Rizhsky L, Liang H, Mittler R. 2002. The combined effect of drought stress and heat shock on gene expression in tobacco. Plant Physiology 130, 1143-1151.

Rizhsky L, Liang H, Shuman J, Shulaev V, Davletova S, Mittler R. 2004. When defense pathways collide. The response of Arabidopsis to a combination of drought and heat stress. Plant Physiology 134, 1683-1696.

Rurek M, Czołpińska M, Pawłowski TA, Krzesiński W, Spiżewski T 2018. Cold and heat stress diversely alter both cauliflower respiration and distinct mitochondrial proteins including OXPHOS components and matrix enzymes. International Journal of Molecular Sciences 19, 877.

Rushton PJ, Somssich IE, Ringler P, Shen QJ. 2010. WRKY transcription factors. Trends in Plant Science 15, 247-258.

Scharf KD, Berberich T, Ebersberger I, Nover L. 2012. The plant heat stress transcription factor (Hsf) family: structure, function and evolution. Biochimica et Biophysica Acta 1819, 104-119.

Schenk PM, Kazan K, Wilson I, Anderson JP, Richmond T, Somerville SC, Manners JM. 2000. Coordinated plant defense responses in Arabidopsis revealed by microarray analysis. Proceedings of the National Academy of Sciences, USA 97, 11655-11660.

Sedaghatmehr M, Mueller-Roeber B, Balazadeh S. 2016. The plastid metalloprotease Fts $\mathrm{H} 6$ and small heat shock protein HSP21 jointly regulate thermomemory in Arabidopsis. Nature Communications 7, 12439.

Sewelam N, Oshima Y, Mitsuda N, Ohme-Takagi M. 2014. A step towards understanding plant responses to multiple environmental stresses: a genome-wide study. Plant, Cell \& Environment 37, 2024-2035.

Shaar-Moshe L, Blumwald E, Peleg Z. 2017. Unique physiological and transcriptional shifts under combinations of salinity, drought, and heat. Plant Physiology 174, 421-434.
Shaik R, Ramakrishna W. 2013. Genes and co-expression modules common to drought and bacterial stress responses in Arabidopsis and rice. PLoS One 8, e77261.

Sormani R, Masclaux-Daubresse C, Daniel-Vedele F, DanieleVedele F, Chardon F. 2011. Transcriptional regulation of ribosome components are determined by stress according to cellular compartments in Arabidopsis thaliana. PLoS One 6, e28070.

Suzuki N, Koussevitzky S, Mittler R, Miller G. 2012. ROS and redox signalling in the response of plants to abiotic stress. Plant, Cell \& Environment 35, 259-270.

Swindell WR, Huebner M, Weber AP. 2007. Transcriptional profiling of Arabidopsis heat shock proteins and transcription factors reveals extensive overlap between heat and non-heat stress response pathways. BMC Genomics 8, 125.

Szabados L, Savouré A. 2010. Proline: a multifunctional amino acid. Trends in Plant Science 15, 89-97.

Taji T, Ohsumi C, luchi S, Seki M, Kasuga M, Kobayashi M, YamaguchiShinozaki K, Shinozakki K. 2002. Important roles of drought- and coldinducible genes for galactinol synthase in stress tolerance in Arabidopsis thaliana. The Plant journal 29, 417-426.

Tolleter D, Hincha DK, Macherel D. 2010. A mitochondrial late embryogenesis abundant protein stabilizes model membranes in the dry state. Biochimica et Biophysica Acta 1798, 1926-1933.

Tunnacliffe A, Wise MJ. 2007. The continuing conundrum of the LEA proteins. Die Naturwissenschaften 94, 791-812.

Verslues PE, Agarwal M, Katiyar-Agarwal S, Zhu J, Zhu JK. 2006. Methods and concepts in quantifying resistance to drought, salt and freezing, abiotic stresses that affect plant water status. The Plant journal 45, 523-539.

Vetter J. 2000. Plant cyanogenic glycosides. Toxicon 38, 11-36.

Xu YH, Liu R, Yan L, Liu ZQ, Jiang SC, Shen YY, Wang XF, Zhang DP. 2012. Light-harvesting chlorophyll a/b-binding proteins are required for stomatal response to abscisic acid in Arabidopsis. Journal of Experimental Botany 63, 1095-1106.

Zelisko A, García-Lorenzo M, Jackowski G, Jansson S, Funk C. 2005. AtFtsH6 is involved in the degradation of the light-harvesting complex II during high-light acclimation and senescence. Proceedings of the National Academy of Sciences, USA 102, 13699-13704.

Zhang H, Sonnewald U. 2017. Differences and commonalities of plant responses to single and combined stresses. The Plant Journal $\mathbf{9 0}$, 839-855. 